Sahr Sana*, Kamelia Boodhoo* and Vladimir Zivkovic

\title{
Production of starch nanoparticles through solvent-antisolvent precipitation in a spinning disc reactor
}

https://doi.org/10.1515/gps-2019-0019

Received September 21, 2018; accepted February 21, 2019.

Abstract: The spinning disc reactor (SDR) uses surface rotation to produce thin film flow with improved mixing and reduced residence times in chemical processing applications. Solvent-antisolvent precipitation is one such process that can benefit from these properties. This study investigates the film hydrodynamics and precipitation of starch nanoparticles by contacting starch dissolved in sodium hydroxide with ethanol as the antisolvent. One objective of this study is to understand how interactions of the disc surface topography (grooved and smooth) with other parameters such as liquid flowrate, antisolvent to solvent flow ratio and disc speed impact the mixing and precipitation processes. Results indicate that an increase in flow rate and rotational speed leads to smaller nanoparticles and narrower size distributions, which is attributed to increased shear and instabilities within the liquid film. It was also observed that an increased antisolvent to solvent ratio caused a reduction in particle size, as increased antisolvent generated higher supersaturation. Results showed that although particle size was not significantly influenced by the disc texture, the size distribution was narrower and higher yields were obtained with the grooved disc surface. The grooved disc therefore offers the opportunity for higher throughput in the solventantisolvent precipitation of starch particles with better product quality.

Keywords: spinning disc reactor; process intensification; solvent-antisolvent precipitation; micromixing; starch nanoparticles

\footnotetext{
* Corresponding authors: Sahr Sana and Kamelia Boodhoo, School of Engineering, Newcastle University, Newcastle upon Tyne, NE1 7RU, UK, e-mail:s.sana@newcastle.ac.uk,kamelia.boodhoo@ newcastle.ac.uk

Vladimir Zivkovic, School of Engineering, Newcastle University, Newcastle upon Tyne, NE1 7RU, UK
}

\section{Introduction}

Precipitation is a process often used for the recovery of solids of high purity from solution [1]. It is a bottom-up process, generating particles from the atomic level as an alternative to milling, an expensive and inefficient process especially in the context of nanoparticle formation. The process of precipitation requires the creation of supersaturation so nucleation and growth may proceed. Supersaturation is the ratio of solute concentration to saturated concentration and is the driving force for precipitation processes. Solvent-antisolvent precipitation allows the creation of supersaturation by lowering equilibrium concentration through addition of the antisolvent [2]. It is a method beneficial for polymorph formation in pharmaceuticals and for systems unstable at changing temperatures [3,4]. Mixing is fundamental in solvent-antisolvent precipitation processes as the generation of uniform supersaturation is highly dependent on sufficient mixing between the antisolvent and the solute/solvent liquids.

The spinning disc reactor (SDR) is a process intensification technology that has been shown to enhance mixing through subjecting the liquid to high centrifugal forces, creating highly sheared, thin and unstable films $[5,6]$. The thin liquid film generated on the surface of disc can exhibit near plug flow characteristics with negligible radial dispersion [7]. Under plug flow conditions the residence time distribution is tightly controlled which allows each reacting species to undergo the same processing experience; for precipitation processes this means narrower particle size distributions (PSD). The SDR has previously been used in many applications typically involving fast and sometimes highly exothermic reactions, including catalytic reactions [8,9], polymerisation reactions $[10,11]$, acid-base neutralisation reactions [12] and reactive precipitations [13-17]. There is not much work on solvent-antisolvent precipitation processes carried out in an SDR, and research is limited to earlier reports on the production of curcumin nanoparticles [18] and the re-crystallisation of an active pharmaceutical ingredient [8]. 
The texture of the disc surface has been shown to affect hydrodynamics, wave formation [19], and residence time distribution [7]. It has been observed that the residence time distribution is narrower with the grooved disc surface [7]. Furthermore, for reactive crystallisation processes, crystals produced on a grooved disc surface have been reported to be smaller in size, with a narrow size distribution than those produced on a smooth disc [14]. Additional use of grooved disc has been reported in processes such as polymerisation [10] and nanoparticle production [20]. Parameters such as flow rate and disc rotational speed have been shown to have further effect on liquid film interaction with the textured discs, influencing the residence time distribution and SDR hydrodynamics [7].

The work presented here concerns the solventantisolvent precipitation of starch nanoparticles. Ethanol has been selected as the antisolvent for this study and sodium hydroxide as the solvent. Starch nanoparticles have many promising applications, ranging from fillers in nanocomposites to carriers in drug delivery systems [21]. Previous methods of generating starch nanoparticles involve acid hydrolysis, enzymatic hydrolysis, and gamma irradiation [22]. Due to these methods belonging to the 'top down' crystallisation category, they often require a lot of energy and result in low yields and broad size distributions [23]. The SDR offers a greener alternative for generating starch nanoparticles with a tighter control of particle size distribution. In spite of an additional power input (typically of up to $2000 \mathrm{~W} / \mathrm{kg}$ of processed fluid) associated with the rotation of the SDR surface and centrifugal acceleration of the fluid, this energy has been shown to be efficiently transmitted to the liquid to enable more efficient micromixing, especially in comparison with a conventional stirred tank reactor [5]. Although it would be beneficial to eliminate the use of solvents altogether, the ethanol antisolvent used in this work has a much lower environmental impact compared to the solvents used in the top-down processes, such as sulfuric acid, and is less toxic and environmentally favourable [24].

The main aim of the current work is to investigate a green production route to producing starch nanoparticles using the solvent-antisolvent precipitation technique in the SDR. The paper first presents the experimental system and methodology used in the study, followed by presentation of the results showing influence of factors such as disc rotational speed, total flow rate and solvent to antisolvent ratio on the size and size distribution of the starch nanoparticles produced. Influence of the smooth and grooved disc textures on the qualities of produced nanoparticles is also investigated and discussed.

\section{Experimental methodology}

Figure 1 shows a schematic of the spinning disc reactor used in this research. The reactor consists of a $30 \mathrm{~cm}$ diameter stainless steel disc (grooved or smooth) encased in a reactor housing which contains the flow of liquid exiting from the edge of the spinning disc. The temperature on the surface of the disc is regulated through water circulation underneath the disc at a maintained temperature of $25^{\circ} \mathrm{C}$. The temperature of the circulation water is controlled in a heating tank where it is held. The flow rates of the individual starch $/ \mathrm{NaOH}$ and ethanol feeds to the disc are provided by Watson Marlow 323E and Watson Marlow 505 S peristaltic pumps respectively which feed the solvent and antisolvent to single point feed distributors situated $2.3 \mathrm{~cm}$ above the centre of the disc.

The reagents sodium hydroxide, 99.8\% absolute ethanol and corn starch were purchased from Fisher Scientific, UK. The solute, starch, was dissolved in $0.5 \mathrm{M}$ sodium hydroxide solution to form a $2 \% \mathrm{w} / \mathrm{v}$ concentration solute/solvent mixture. A 20\% w/w concentration (based on starch weight) of Tween 80 was used as the surfactant to prevent agglomeration of starch.

The conditions that were studied in the SDR included disc rotational speed (400, 800 and $1200 \mathrm{rpm})$, total flow rate $(6,12$ and $18 \mathrm{~mL} / \mathrm{s})$, and antisolvent to solvent ratio (1:1, 5:1, 9:1 on a volume basis). The results presented here are for experiments conducted on a stainless steel grooved disc with 8 concentric grooves and a stainless steel smooth disc as shown in Figure 2.

Samples of starch nanoparticles from the disc were analysed using Dynamic Light Scattering (DLS - Mode Nano ZS Malvern instruments, UK) technology to obtain particle size and size distributions. The size measurements were all carried out at $25^{\circ} \mathrm{C}$. Each sample measurement was performed in triplicates, with the average of the size distribution measurements and the mean peak sizes being reported for the primary peak. Although in many cases a smaller second peak corresponding to agglomeration is present, the focus here is on the first peak for analysing the

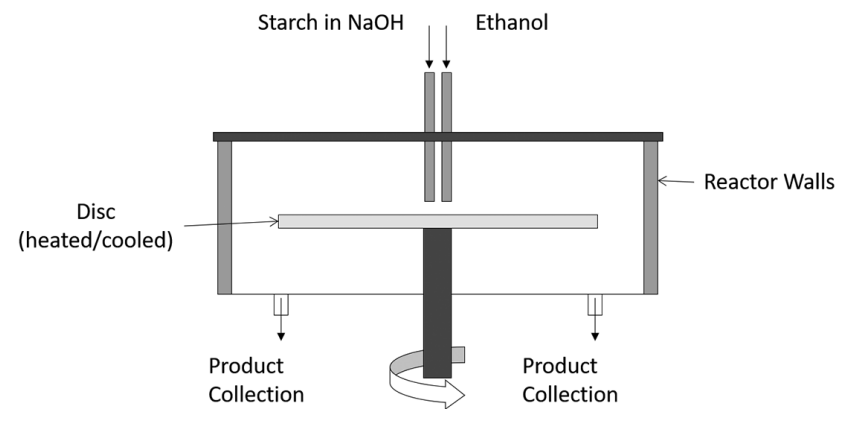

Figure 1: Schematic of SDR. 
size of the particles, and for this reason the agglomeration peak will be ignored, unless specified. A typical PSD showing both peaks is shown in Figure 3. The width of the size distribution is represented by a Polydispersity Index (PdI) value. Again, this has been calculated for the single peak of interest, and not for the entire distribution, unless specifically stated.

Transmission electron microscopy (Philips CM100 $100 \mathrm{kV}$ TEM, FEI) of the samples was also performed for a realistic quantification of the actual particle size as well as for studying the morphology and the presence of any agglomeration amongst the nanoparticles. Images were taken with an Optronics AMT40 CCD camera, $1824 \times 1824$ pixel (Deben UK) using 400 mesh copper grids (Gilder Grids UK) with negative staining.

To estimate particle yield, the particles were subjected to centrifugation at $1000 \mathrm{rpm}$ for 2 minutes to separate

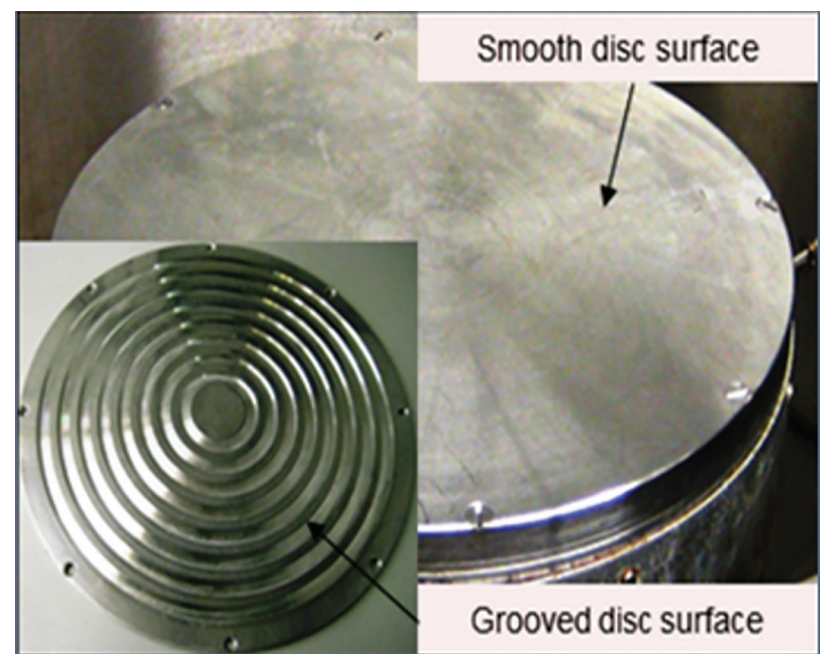

Figure 2: The grooved and smooth disc surfaces used in SDR experiments.

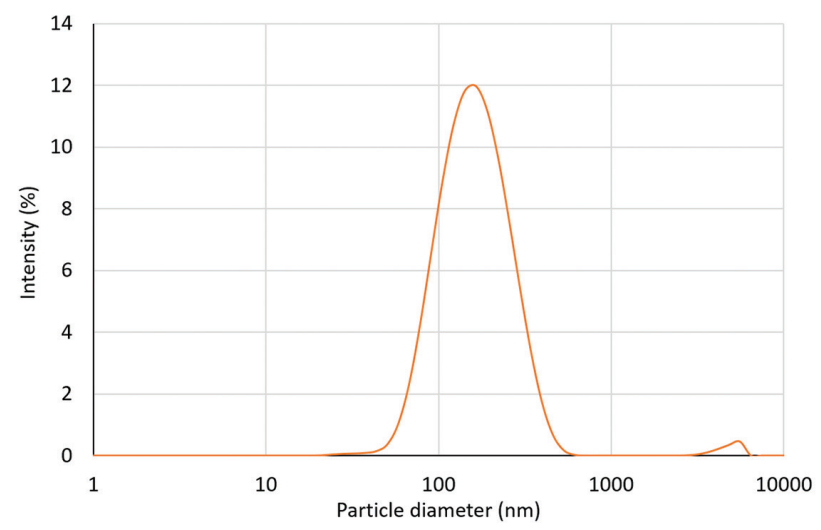

Figure 3: Overall PSD showing the primary peak and an agglomeration peak. and remove the supernatant. This was repeated 5 times and washed with ethanol to remove the $\mathrm{NaOH}$ and water in between centrifuging. The particles were then left to dry under vacuum at $35^{\circ} \mathrm{C}$. The particles were weighed to estimate yield using the following equation:

$$
\text { Yield }(\%)=\frac{\text { Mass of dried product }}{\text { Mass of initial starchin } 2 \% w / v \text { solution }} \times 100
$$

\section{Results}

\subsection{Effect of total flow rate on nanoparticle size distribution}

Figure 4 shows the effect of total flow rate on starch nanoparticle size distribution at constant disc speed of $1200 \mathrm{rpm}$ and a 9:1 antisolvent to solvent ratio on the grooved disc. The figure shows that an increase in total flow rate decreases both the average size of particles and the size distribution width, which is evident from the peak at $175 \mathrm{~nm}$ and a polydispersity index of 0.204 for $18 \mathrm{~mL} / \mathrm{s}$ flow rate in comparison to the size and PdI value obtained at $6 \mathrm{~mL} / \mathrm{s}$ which are $248 \mathrm{~nm}$ and 0.302 respectively, as can be seen in Figure 4a. Figure 4b shows how the flow rate affects mean particle size and PdI value. There is a general decline in particle size and PdI value as flow rate increases. However, between $12 \mathrm{~mL} / \mathrm{s}$ and $18 \mathrm{~mL} / \mathrm{s}$ no further reduction in particle size is observed as flow rate increases, which is a possible result of agglomeration of smaller particles as more particles are generated at the higher flow rate. Similar trends are observed at all disc speeds and antisolvent to solvent ratios. The TEM images in Figure 5 presents a comparison between particles produced with a flow rate of 6 and $18 \mathrm{~mL} / \mathrm{s}$. This analysis confirms that higher flow rate produced smaller sized particles. Average size of particles at $18 \mathrm{~mL} / \mathrm{s}$ form TEM analysis was found to be $13.3 \mathrm{~nm}$, whereas

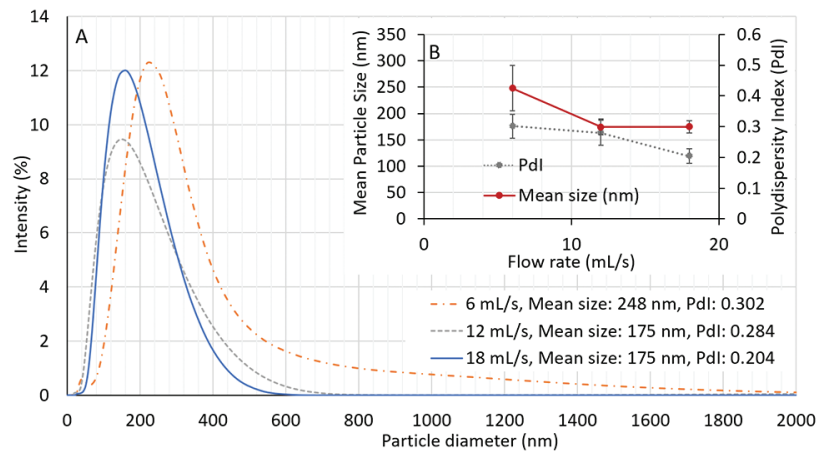

Figure 4: Effect of flow rate at $1200 \mathrm{rpm}, 9: 1$ antisolvent to solvent ratio, on the grooved disc (a) PSD, (b) plot of mean particle size and Pdl. 


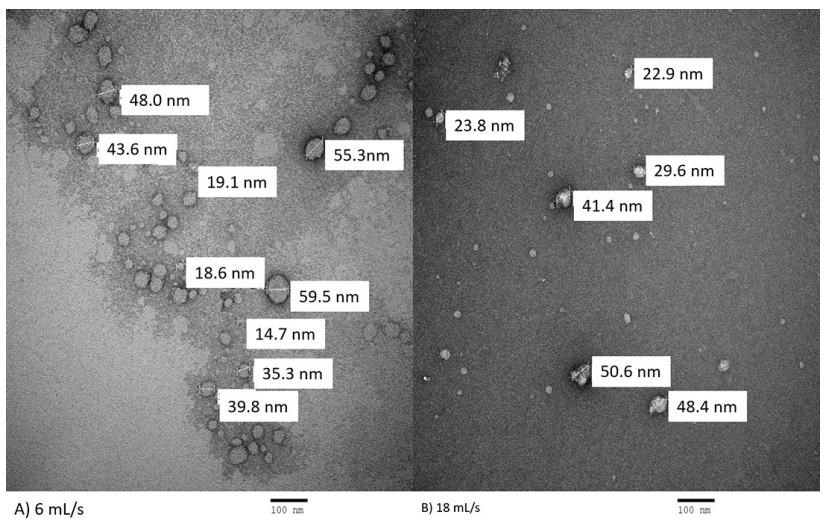

Figure 5: TEM images for (a) $6 \mathrm{~mL} / \mathrm{s}$, and (b) $18 \mathrm{~mL} / \mathrm{s}$ at $1200 \mathrm{rpm}$ and 9:1 ratio.

at $6 \mathrm{~mL} / \mathrm{s}$ the average size was higher at $30.3 \mathrm{~nm}$. There is also no evidence of significant agglomeration between the starch nanoparticles taking place at either flow rates. It should be noted that the difference in particle sizes from DLS and TEM is a result of larger particles scattering more light in the DLS technique which shifts the peaks towards the larger end of the size distribution, whereas the TEM shows the true particle size [25].

The increase in instabilities within the film enhances micromixing between the solvent and antisolvent which aids in the production of uniformly high supersaturation, causing nucleation of starch nanoparticles. The spinning disc reactor does not increase supersaturation itself, as supersaturation and nucleation processes are independent of reactor geometry [26]. However, it does prevent high local supersaturation through effective mixing of the solvent and antisolvent streams flowing on the disc, thereby ensuring uniform supersaturation across the film layer. Within this ideal uniform mixing environment, more successful collisions between molecules can occur to form the critical nucleus required for particle nucleation to take place, thus increasing nucleation rate in this way. An increase in nucleation rate means that supersaturation is consumed primarily by nucleation which contributes to the formation of reduced particle size [27]. Growth of particles and even agglomeration may be further reduced as the residence time decreases with an increase in flow rate, according to Eq. 2, where, $t_{\text {res }}$ is the residence time on the SDR, $v$ is the kinematic viscosity of the liquid, $\omega$ is the angular velocity of the SDR, $Q_{T}$ is the total flow rate, and $r_{o}$ and $r_{i}$ are the radial distances at exit and entry points respectively from the centre of the disc.

$$
t_{r e s}=\left(\frac{81 \pi^{2} v}{16 \omega^{2} Q_{T}^{2}}\right)^{1 / 3}\left(r_{0}^{4 / 3}-r_{i}^{4 / 3}\right)
$$

The decrease in particle size distribution width at increasing flow rates as indicated by the lower PdI values suggest superior transverse mixing occurs across the film thickness whilst reduced radial mixing occurs leading to a narrow residence time distribution, and thus a narrow particle size distribution. This effect of flowrate on residence time distribution has been quantitatively demonstrated in an earlier study [7]. The results obtained are in agreement with prior solvent-antisolvent precipitation work done on the SDR, where increasing flow rate has resulted in smaller sized particles [18].

\subsubsection{Effect of disc rotational speed on nanoparticle size distribution}

A similar effect as flow rate has been observed for disc rotational speeds investigated at 400, 800 and $1200 \mathrm{rpm}$, presented in the PSD profiles in Figure 6a. At higher disc speeds, there is a reduction in both mean particle size and PdI as shown in Figure 6b. An increase in disc rotational speed increases mixing and contact between antisolvent and starch solution due to reduced film thickness and more induced turbulence within the film, which leads to an increase in nucleation rate and decrease in particle growth. Furthermore, the disc speed has also been shown to influence plug flow behaviour [7], with flow approaching plug flow at higher disc rotational speeds and at high flow rates. Plug flow conditions aid in mixing between the solvent and antisolvent as well as reduce backmixing on the disc, decreasing the variation between particles thus leading to a much narrower size distribution. In addition to this, higher rotational speeds and flow rates have been associated with increased shear of the film and an increase in ripples on the film surface [28]. Consequently,

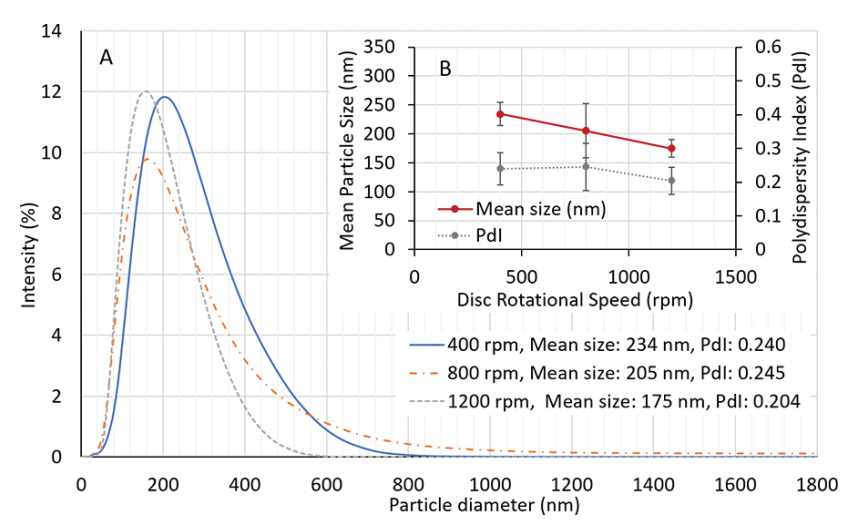

Figure 6: Effect of disc rotational speed at 9:1 antisolvent to solvent ratio and $18 \mathrm{~mL} / \mathrm{s}$ flow rate on the grooved disc, (a) PSD, (b) plot of mean particle size and PdI. 


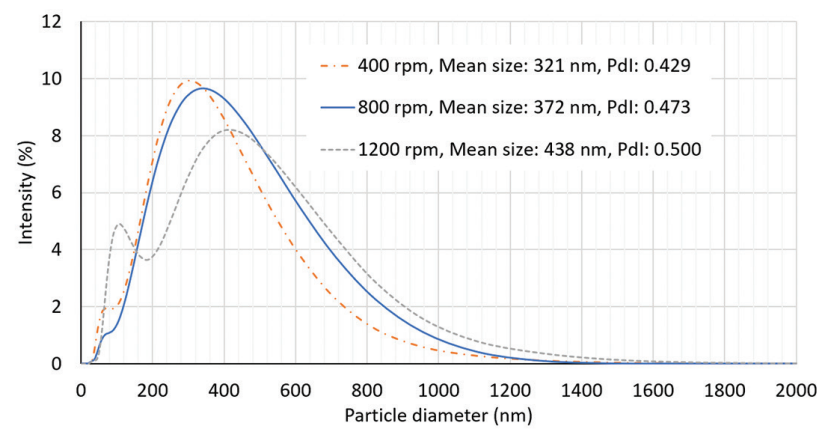

Figure 7: Effect of disc rotational speed on PSD of starch nanoparticles at 1:1 antisolvent to solvent ratio and $18 \mathrm{~mL} / \mathrm{s}$.

the increased instabilities within the liquid film lead to enhanced mixing between the solvent and antisolvent streams. These results agree with previous work done on solvent-antisolvent precipitation, where an increase in rotational speed resulted in smaller particles [18].

It was, however, observed that the combination of low antisolvent to solvent ratio of 1:1 and a high flow rate of $18 \mathrm{~mL} / \mathrm{s}$ on the grooved surface resulted in the production of larger and agglomerated particles. This is evident in the size distribution seen in Figure 7, showing the sharp increase in mean particle size at 1:1 at $18 \mathrm{~mL} / \mathrm{s}$ compared to the corresponding values at 9:1 antisolvent to solvent ratio and $18 \mathrm{~mL} / \mathrm{s}$ shown in Figure 4. The particle size increases with further increase in disc speed. An explanation for this would be poor mixing between the two inlet streams at much higher solvent flow rates, or more specifically the occurrence of back-mixing in the region of the inlet tubes, causing precipitation to take place within the central region of the disc. In effect, when the flow rates of the two inlet streams are similar as when the antisolvent to solvent ratio is 1:1, it is more challenging to incorporate the large quantity of solvent/solute stream within the antisolvent stream, more so under the less than ideal mixing conditions in the inner zones of the disc. This phenomenon has also been experienced in past studies $[18,29]$. If such low antisolvent to solvent ratios are to be used, it may be more advantageous to introduce the antisolvent stream onto the spinning disc away from the central region where more stable and improved hydrodynamic conditions prevail [14].

\subsubsection{Effect of antisolvent to solvent ratio on nanoparticle size distribution}

Figure 8a shows the particle size distribution profiles at selected antisolvent to solvent ratios of 1:1, 5:1 and 9:1 at constant conditions of $12 \mathrm{~mL} / \mathrm{s}$ total flow rate and a disc rotational speed of $1200 \mathrm{rpm}$. An antisolvent to solvent ratio of 9:1 shows a peak of lowest mean size at $175 \mathrm{~nm}$ whereas the PdI value increases with the ratio as shown in Figure 8b. An increase in antisolvent to solvent ratio causes an increase in supersaturation through reduction of starch solubility. The generated supersaturation is then consumed by nucleation processes, generating smaller sized particles. The increase in PdI, on the other hand is likely to be a consequence of greater chances of agglomeration between the small size primary nuclei occurring as a result of more particles of smaller size being produced at the highest ratio. However, the overall PdI, which is a combination of the PdIs of the main peak, and a much smaller, less intense peak of higher particle size corresponding to agglomerated particles, as depicted in Figure 9, shows that there is more overall agglomeration at the low antisolvent to solvent ratio of 1:1. Agglomeration at 1:1 ratio may be the result of the entrapment of solvent between particles in close proximity to each other as incorporation of the higher amount of solvent/solute stream within the antisolvent stream becomes more

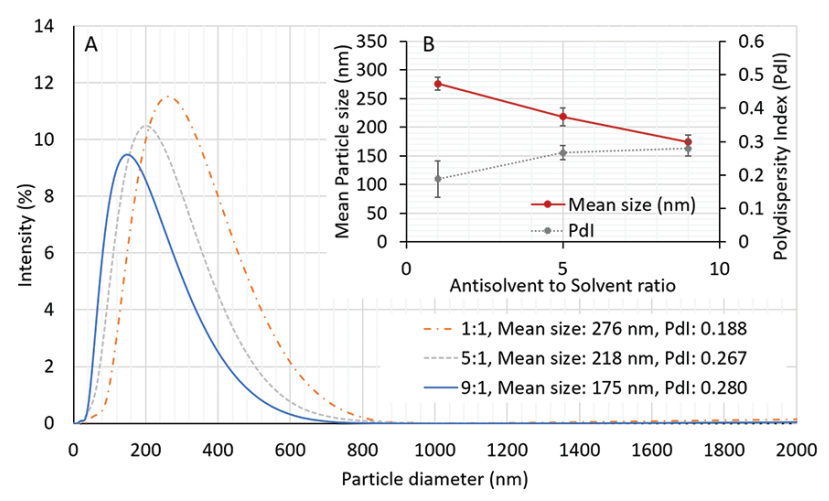

Figure 8: Effect of antisolvent to solvent ratio at $12 \mathrm{~mL} / \mathrm{s}$ and $1200 \mathrm{rpm}$ on the grooved disc, (a) PSD, (b) plot of mean particle size and PdI.

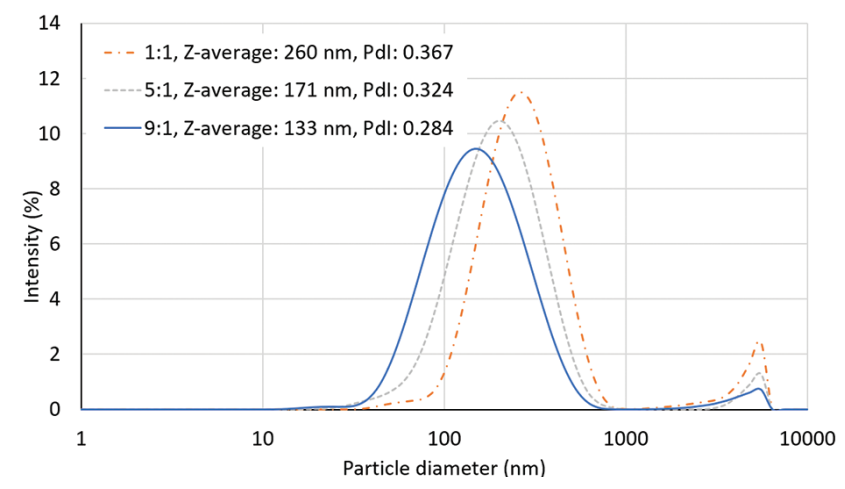

Figure 9: Overall PSD showing effect of antisolvent to solvent ratio at $12 \mathrm{~mL} / \mathrm{s}$ and $1200 \mathrm{rpm}$. 
difficult, as discussed in Section 3.1.1 earlier. Furthermore, as particles formed at 1:1 ratio are larger in size, the coverage by the surfactant of the larger particles may not be sufficient with the same concentration of surfactant used for all experimental conditions. Hence greater free surface is available on larger particles for agglomeration with surrounding particles. Table 1 presents the individual and overall PdI values for the 3 antisolvent to solvent ratios, along with the $\mathrm{z}$-averages. The $\mathrm{z}$-average is a cumulant size representing the average of the 2 peaks present in the PSD.

TEM images in Figure 10 show the starch nanoparticles produced using 9:1, 5:1 and 1:1 ratios at $18 \mathrm{~mL} / \mathrm{s}$ and $1200 \mathrm{rpm}$. The images agree with the particle size distributions obtained using the DLS Zetasizer. In particular the presence of agglomerates at 9:1 ratio can be seen in Figure 10. Furthermore, the images show a reduction in particle size as the antisolvent to solvent ratio increases. This can be backed up by the size distribution in Figure 11, developed using the TEM images.

Table 1: Particle sizes and PdI values for different antisolvent to solvent ratios at $12 \mathrm{~mL} / \mathrm{s}$ and $1200 \mathrm{rpm}$.

\begin{tabular}{lrrrrr}
\hline $\begin{array}{l}\text { Antisolvent } \\
\text { to solvent } \\
\text { ratio }\end{array}$ & $\begin{array}{r}\text { Mean peak 1 } \\
(\mathrm{nm})\end{array}$ & $\begin{array}{r}\text { Mean peak 2 } \\
(\mathrm{nm})\end{array}$ & $\begin{array}{r}\text { Z-average } \\
(\mathrm{nm})\end{array}$ & $\begin{array}{r}\text { Pdl of } \\
\text { peak 1 }\end{array}$ & $\begin{array}{r}\text { Pdl } \\
\text { overall }\end{array}$ \\
\hline $1: 1$ & & & & & \\
\hline $5: 1$ & 276.26 & 4813.33 & 259.57 & 0.188 & 0.367 \\
$9: 1$ & 218.43 & 4952.33 & 171.27 & 0.267 & 0.324 \\
\hline & 174.49 & 3789.00 & 133.35 & 0.280 & 0.284 \\
\hline
\end{tabular}

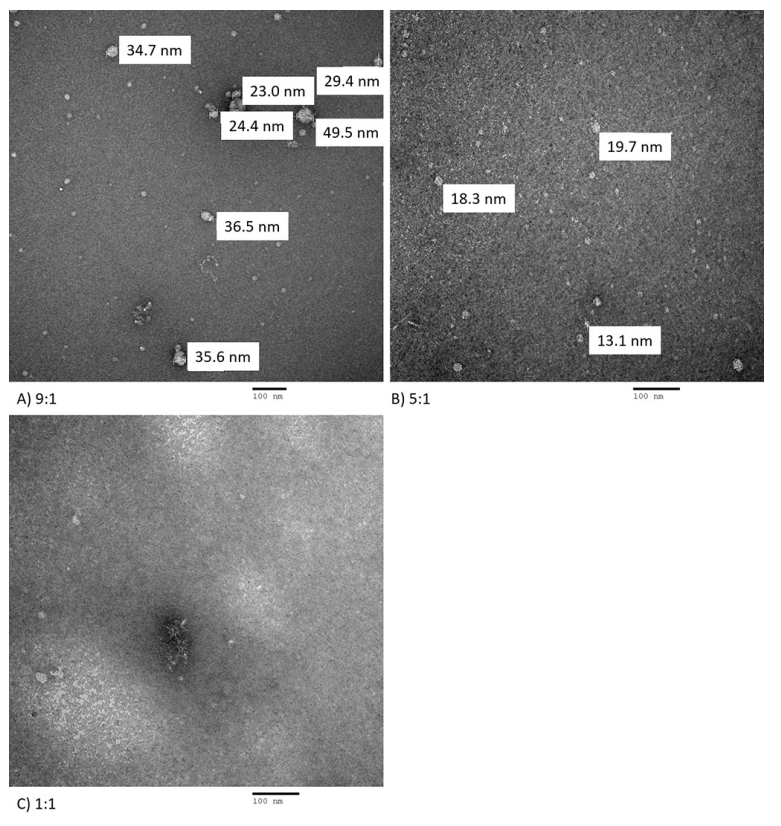

Figure 10: TEM images for (a) 9:1, (b) 5:1 and (c) 1:1 antisolvent to solventratios at $18 \mathrm{~mL} / \mathrm{s}$ and $1200 \mathrm{rpm}$.

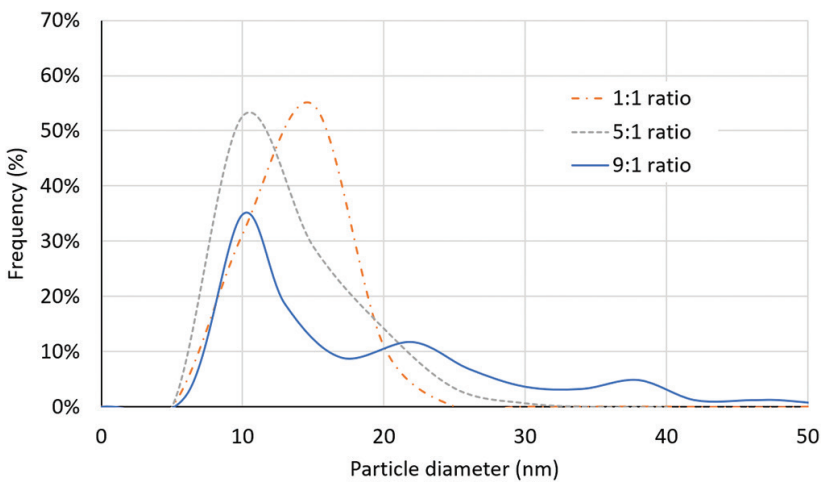

Figure 11: PSD developed from TEM images in Figure 10, showing effect of antisolvent to solvent ratio at $18 \mathrm{~mL} / \mathrm{s}$ and $1200 \mathrm{rpm}$.

Although previous work focusing on solvent-antisolvent precipitation of starch nanoparticles has been carried out in a semi-batch system, it has been limited to 1:1 ratios of antisolvent to solvent and resulting particle size produced have been between 130 to $140 \mathrm{~nm}$ [23]. However, in this present study an average particle size of $11.45 \mathrm{~nm}$ has been obtained for 1:1 antisolvent to solvent ratio at $18 \mathrm{~mL} / \mathrm{s}$ and $1200 \mathrm{rpm}$. For such a small particle size to be produced in a semi-batch process would require further downstream processing such as milling which could consume a significant amount of energy.

\subsection{Comparison between smooth and grooved disc surfaces}

Figure 12 compares the smooth and grooved discs for the production of starch nanoparticles. The trend followed in the smooth disc is similar to that observed on the grooved disc for the conditions studied, and for reasons discussed earlier.

Figure 12a shows the effect of increasing flow rate, which suggests the production of smaller sized starch nanoparticles on the grooved disc up until $12 \mathrm{~mL} / \mathrm{s}$. However, after that there is not much more of a reduction in particle size as the flow rate is increased to $18 \mathrm{~mL} / \mathrm{s}$. On the other hand, for the smooth disc the particle size continues to decrease to produce slightly smaller sized particles than on grooved surface. This is also true for the effect of rotational speed presented in Figure 12b. For the conditions of $18 \mathrm{~mL} / \mathrm{s}$ flow rate, 9:1 ratio and 1200 rpm disc speed, the PSD in Figure 13 reveals a lower particle size on smooth disc surface, however the size distribution is narrower with the grooved disc, resulting in a lower overall PdI. The TEM images in Figure 14 for these conditions confirm the presence of more agglomeration amongst particles produced on the smooth disc. 

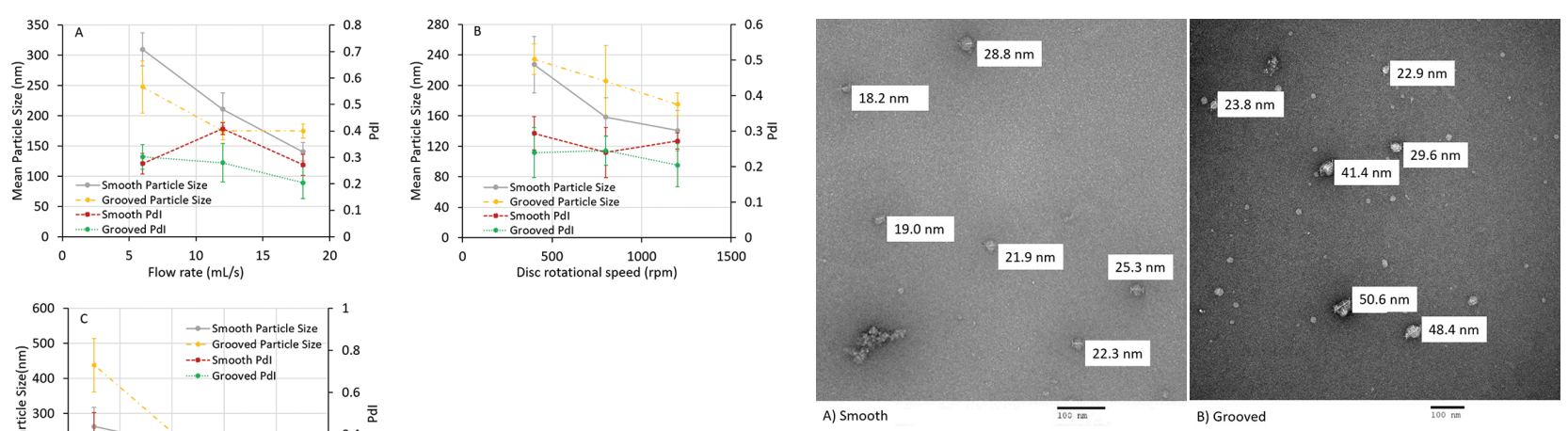

Figure 14: TEM image at $18 \mathrm{~mL} / \mathrm{s}, 9: 1$ ratio and $1200 \mathrm{rpm}$ for (a) smooth and (b) grooved surfaces.

Figure 12: Comparison between smooth and grooved disc for the effect of (a) flow rate (1200 rpm, 9:1 ratio), (b) disc rotational speed $(18 \mathrm{~mL} / \mathrm{s}, 9: 1$ ratio), (c) antisolvent to solvent ratio (1200 rpm, $18 \mathrm{~mL} / \mathrm{s})$.

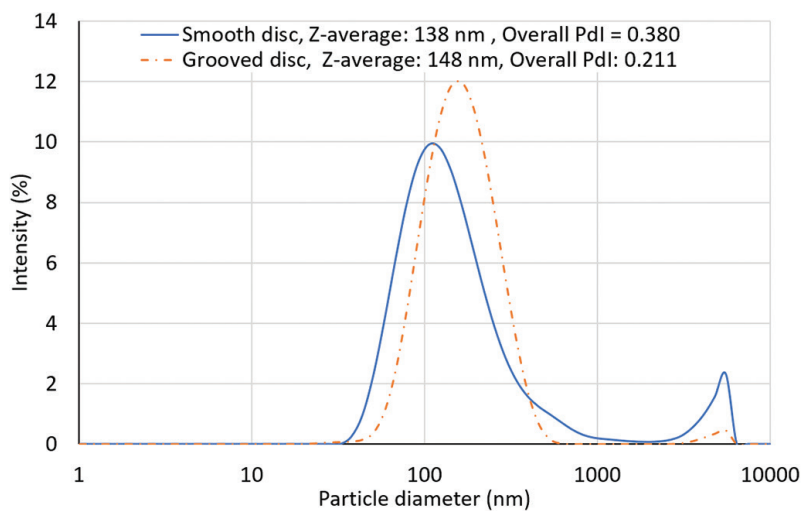

Figure 13: PSD comparing smooth and grooved disc surfaces at 18 $\mathrm{mL} / \mathrm{s}, 9: 1$ ratio and $1200 \mathrm{rpm}$.

Figure 12c shows the effect of antisolvent to solvent ratio for smooth and grooved disc surfaces. There is not a significant difference between particle sizes obtained on the smooth and grooved discs, particularly at the higher ratios of 5:1 and 9:1. This is most likely due to supersaturation being a more important factor than surface texture, and the supersaturation generated is the same for both surfaces at a given antisolvent to solvent ratio.

The effect of the texture of the disc surface on mean particle size and its interactions with total flow rate, disc rotational speed and antisolvent to solvent ratio are presented in Figure 15, as well as the interactions between other parameters. The interactions plot summarises and agrees with the results so far. The interaction between total flow rate and the antisolvent to solvent ratio especially at high flow rates and low antisolvent to solvent ratio is particularly evidenced by the reverse trend observed at these values Generally, when comparing the particle sizes obtained

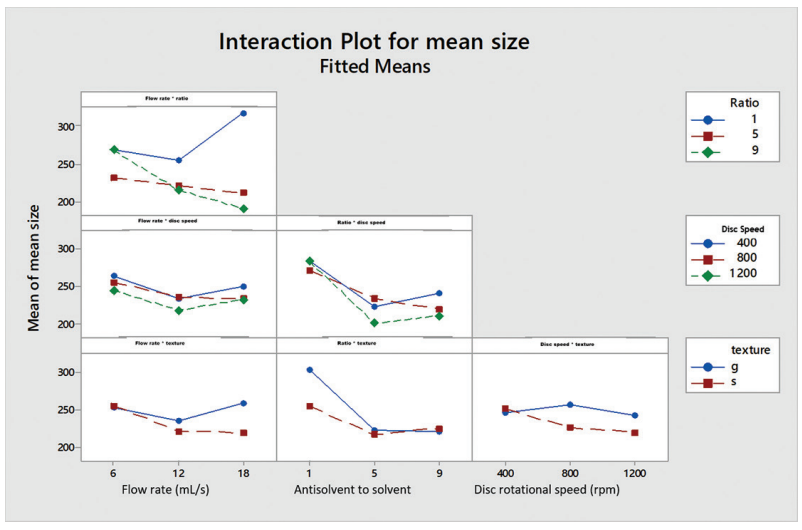

Figure 15: Interactions plot showing effect of interactions between parameters on particle size obtained from the DLS.

on the smooth and grooved disc for the same conditions, the particles produced on the smooth disc are smaller. However, looking at the error bars in Figure 12, they tend to overlap in a few areas which suggests that the effect of disc surface on particle size is not as profound as it is on the width of the particle size distribution.

The lower PdI on the grooved disc is the consequence of increased plug flow, associated with the narrower residence time distribution. It is a result of the textured surface generating turbulent eddies within the liquid film as it travels along the grooved surface. Furthermore, a grooved surface also promotes surface wetting and reduces the chances of film breakdown when compared to a smooth surface [7]. All these hydrodynamic effects prevalent on the grooved disc in contrast to the smooth disc provide the best conditions for controlling the PdI, thus resulting in better quality starch particles upon precipitation.

\subsubsection{Yield}

Figure 16 shows the effects of flow rate, disc speed, and antisolvent to solvent ratio on particle yield for both 

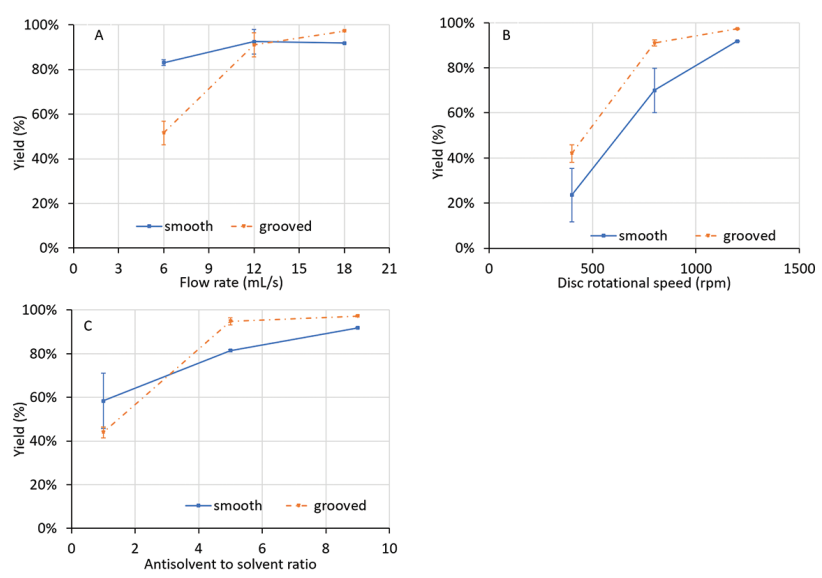

Figure 16: Yields for smooth and grooved discs for the effect of (a) flow rate (1200 rpm, 9:1 ratio), (b) disc rotational speed $(18 \mathrm{~mL} / \mathrm{s}$, 9:1 ratio), (c) antisolvent to solvent ratio (1200 rpm, $18 \mathrm{~mL} / \mathrm{s}$ ).

smooth and grooved disc surfaces. As discussed earlier, increased flow rates, higher disc speeds and increased proportion of antisolvent promote nucleation due to better mixing conditions. This generally translates into higher yields of particles under these conditions, especially so on the grooved disc.

\section{Conclusion}

Starch nanoparticles were produced through a solventantisolvent precipitation process in the spinning disc reactor using a grooved and a smooth disc surface. The impacts of operating conditions such as total flow rate, antisolvent to solvent ratio and disc rotational speed were investigated. It was observed that an increase in flow rate, antisolvent to solvent ratio and disc speed all caused a reduction in particle size. However, an increased antisolvent flow rate at a total flow rate of $18 \mathrm{~mL} / \mathrm{s}$ and 1:1 antisolvent to solvent ratio on the grooved surface was an exception to this due to possible back-mixing as the liquid bounces off the grooves on introduction of the equally high flows of both feeds in the inner regions of the disc. Yield experiments also showed a similar trend of increases in flow rate, disc speed and antisolvent to solvent ratio resulting in higher yields, more so on the grooved disc. It may be deduced that disc speed and total flow rate increase shear and instabilities within the liquid film, enhancing mixing between solvent and antisolvent, thus producing smaller sized starch nanoparticles with narrow PSDs. In addition to this, the increased antisolvent to solvent ratio decreases solubility and promotes nucleation through increased supersaturation.
Comparisons between the smooth and grooved surfaces were made to investigate their impact on particle size, PdI and yield. It was noticed that although the smooth surface resulted in slightly smaller particles in some cases, the smooth surface of the disc did not have an extreme effect on particle size, and thus it would be reasonable to conclude that the disc surface does not significantly affect the mean size of the starch nanoparticles. In contrast, the disc surface was found to have a more significant impact on PdI value, caused by increased plug flow on the grooved disc, which resulted in narrower size distributions.

In this work, we have demonstrated the processing advantages of generating smaller, more uniformly sized particles at relatively high yields in a continuous, intensified SDR technology. Combined with the use of ethanol as a greener solvent than the toxic and harmful solvents typically employed in the precipitation of starch nanoparticles, process intensification in the SDR offers the opportunity for greener, more efficient processing to be developed in antisolvent-solvent precipitation applications.

\section{References}

[1] Mullin J.W., Crystallization (4th ed.). Butterworth-Heinemann, Oxford, 2001.

[2] Jones A.G., Crystallization principles and techniques. In: Jones A.G. (Ed.), Crystallization Process Systems. ButterworthHeinemann, Oxford, 2002.

[3] Takiyama H., Minamisono T., Osada Y., Matsuoka M., Operation design for controlling polymorphism in the anti-solvent crystallization by using ternary phase diagram. Chem. Eng. Res. Des., 2010, 88, 1242-1247.

[4] Doki N., Kubota N., Yokota M., Kimura S., Sasaki S.J., Production of sodium chloride crystals of uni-modal size distribution by batch dilution crystallization. Chem. Eng. Jpn., 2002, 35, 1099-1104.

[5] Boodhoo K.V.K., Al-Hengari S.R., Micromixing characteristics in a small-scale spinning disk reactor. Chem. Eng. Technol., 2012, 35, 1229-1237.

[6] Jacobsen N.C., Hinrichsen O., Micromixing efficiency of a spinning disk reactor. Ind. Eng. Chem. Res., 2012, 51, 11643-11652.

[7] Mohammadi S., Boodhoo K.V.K., Online conductivity measurement of residence time distribution of thin film flow in the spinning disc reactor. Chem. Eng. J., 2012, 207-208, 885-894.

[8] Oxley P., Brechtelsbauer C., Ricard F., Lewis N., Ramshaw C., Evaluation of spinning disk reactor technology for the manufacture of pharmaceuticals. Ind. Eng. Chem. Res., 2000, 39, 2175-2182.

[9] Vicevic M., Boodhoo K.V.K., Scott K., Catalytic isomerisation of $\alpha$-pinene oxide to campholenic aldehyde using silica-supported 
zinc triflate catalysts: I. Kinetic and thermodynamic studies. Chem. Eng. J., 2007, 133(1-3), 43-57.

[10] Boodhoo K.V.K., Jachuck R.J., Process intensification: spinning disk reactor for styrene polymerisation. Appl. Therm. Eng., 2000, 20, 1127-1146.

[11] Dobie C.G., Vicevic M., Boodhoo K.V.K., An evaluation of the effectiveness of continuous thin film processing in a spinning disc reactor for bulk free-radical photo-copolymerisation. Chem. Eng. Process., 2013, 71, 97-106.

[12] Ghiasy D., Tham M.T., Boodhoo K.V.K., Control of a spinning disc reactor: an experimental study. Ind. Eng. Chem. Res., 2013, 52(47), 16832-16841.

[13] Cafiero L.M., Baffi G., Chianese A., Jachuck R.J.J., Process intensification: precipitation of barium sulfate using a spinning disk reactor. Ind. Eng. Chem. Res., 2002, 41, 5240-5246.

[14] Mohammadi S., Harvey A., Boodhoo K.V.K., Synthesis of $\mathrm{TiO}_{2}$ nanoparticles in a spinning disc reactor. Chem. Eng. J., 2014, 258, 171-184.

[15] Tai C.Y., Tai C.T., Chang M.H., Liu H.S., Synthesis of magnesium hydroxide and oxide nanoparticles using a spinning disk reactor. Ind. Eng. Chem. Res., 2007, 46, 5536-5541.

[16] Chin S.F., Iyer K.S., Raston C.L., Saunders M., Size selective synthesis of superparamagnetic nanoparticles in thin fluids under continuous flow conditions. Adv. Funct. Mater., 2008, 18, 922-927.

[17] Ahoba-Sam C., Boodhoo K.V.K., Olsbye U., Jens K.J., Tailoring Cu Nanoparticle Catalyst for Methanol Synthesis Using the Spinning Disk Reactor. Materials, 2018, 11, 154.

[18] Khan W.H., Rathod V.K., Process intensification approach for preparation of curcumin nanoparticles via solvent-nonsolvent nanoprecipitation using spinning disc reactor. Chem. Eng. Process., 2014, 80, 1-10.

[19] Jachuck R.J.J., Ramshaw C., Process intensification: Heat transfer characteristics of tailored rotating surfaces. Heat Recov. Syst. CHP, 1994, 14, 475-491.
[20] Iyer K.S., Raston C.L., Saunders M., Continuous flow nanotechnology: manipulating the size, shape, agglomeration, defects and phases of silver nano-particles. Lab on a Chip, 2007, 7, 1800-1805.

[21] Le Corre D., Bras J., Dufresne A., Starch nanoparticles: a review. Biomacromolecules, 2010, 11, 1139-1153.

[22] Kim H.Y., Park S.S., Lim S.T., Preparation, characterization and utilization of starch nanoparticles. Colloids Surf. B., 2015, 126, 607-620.

[23] Hebeish A., El-Rafie M.H., EL-Sheikh M.A., El-Naggar M.E., Ultra-Fine Characteristics of Starch Nanoparticles Prepared Using Native Starch With and Without Surfactant. J. Inorg. Organomet. Polym., 2014, 24, 515-524.

[24] Capello C., Fischer U., Hungerbühler K., What is a green solvent? A comprehensive framework for the environmental assessment of solvents. Green Chem., 2007, 9, 927-934.

[25] Hagendorfer H., Kaegi R., Parlinska M., Sinnet B., Ludwig C., Ulrich A., Characterization of silver nanoparticle products using asymmetric flow field flow fractionation with a multidetector approac - a comparison to transmission electron microscopy and batch dynamic light scattering. Anal Chem., 2012, 84, 2678-2685.

[26] McCarthy E.D., Dunk W.A.E., Boodhoo K.V.K., Application of an intensified narrow channel reactor to the aqueous phase precipitation of barium sulphate. J. Colloid Interface Sci., 2007, 72-87.

[27] Tung H.H., Paul E.L., Midler M., McCauley J.A., Crystallization of organic compounds. John Wiley \& Sons, Inc., 2008.

[28] Ozar B., Cetegen B.M., Faghri A., Experiments on the flow of a thin liquid film over a horizontal stationary and rotating disk surface. Exp. Fluids., 2003, 34, 556-565.

[29] Chen Y.S., Tai C.Y.D., Chang M.H., Liu H.S., Characteristics of micromixing in a rotating packed bed. J. Chin. Inst. Chem. Eng., 2006, 37, 63-69. 